\title{
Sequestration and Carbon Storage Potential of Tropical Forest Reserve and Tree Species Located within Benue State of Nigeria
}

\author{
Ishaq S. Eneji ${ }^{*}$, Ofoegbu Obinna1, Emmanuel T. Azua ${ }^{2}$ \\ ${ }^{1}$ Department of Chemistry College of Science, Federal University of Agriculture, Makurdi, Benue State, Nigeria \\ ${ }^{2}$ Department of Biological Sciences, College of Science, Federal University of Agriculture, Makurdi, Benue State, \\ Nigeria \\ Email: ${ }^{*}$ eneji3@yahoo.com
}

Received December 2013

\begin{abstract}
Carbon sequestration potential of tree species within forest reserves and other sites in Makurdi Benue state of Nigeria was investigated using non-destructive Walkley-Black technique. The result indicates that $P$. americana has the highest $\mathrm{CO}_{2}$ sequestration potential $(125,916.7 \mathrm{~kg})$, while $T$. grandis $(10.4 \mathrm{~kg})$ and $D$. regia $(26.1 \mathrm{~kg})$ were the least. The study also shows that trees (T. grandis, $S$. actinophylla and $P$. americana) with thick vegetation, broad and clustered leaves were found to be better $\mathrm{CO}_{2}$ sequesters. The relationship between the tree height and amount of $\mathrm{CO}_{2}$ sequestered gave a regression equation of $y=67898 x+9509$ with $R^{2}=0.266$, indicating insignificant variations existing between tree height and $\mathrm{CO}_{2}$ sequestration at $\mathrm{P}>0.05$. Variations however existed between tree dominance and $\mathrm{CO}_{2}$ sequestration among trees investigated. This finding strategically positions Benue tropical forest in line for carbon credit financing while substantiating the importance of preserving our indigenous forest and tree species.
\end{abstract}

\section{Keywords}

Benue; Carbon Credit; Tropical Forest; Bio-Diversity; Sequestration Potential; Bio-Sink

\section{Introduction}

The importance of forests in extenuating climate change has led many countries to study the carbon budgets and carbon sequestration of their forests resource. The total global potential for afforestation and reforestation activities for the period 1995-2050 is estimated to be an average of 1.35Pg C per year, of which 70\% possibly will occur in the tropics (IPCC, 2001).

Nigeria had sixty (60) million hectares of forest as area as at 1897 and it is established that this has declined during the 1990s at an estimated annual rate of $2.6 \%$ or more ( $\leq 398,000$ hectares/year) (Oguntala, 1995; FAO, 2005). Observed causative agents were, agricultural expansion, encroachment, over-harvesting, bush burning, illegal harvesting and de-reservations. The Federal Department of Forestry postulated that the annual diminution 
rate may be as high as $3.5 \%$, noting that the forest estate of Nigeria is being highly depleted with the Sahara Desert encroaching southward at a rate of about one kilometer per year. This phenomenon appearing very docile poses great natural risk and creates a disaster laden picture for the country, this makes mitigation and related containment activities very imperative.

Plants' propagative and vegetative mechanisms are known to contribute to the control of the experienced and increasing carbon dioxide emission in the environment. The utilization of available atmospheric $\mathrm{CO}_{2}$ in photosynthetic process provides a natural sink for the excess carbon dioxide generated by anthropogenic activities. It is known that global emission of carbon dioxide has increased by 18\% reaching its first peak level after 1750 . An up surge level increase per year of $1.5 \mathrm{ppb}$ in 1990-2000, $2 \mathrm{ppb}$ in 2001-2009 and 2.3 ppb in 2009-2010 the highest in recent decades (Chavan and Rasal, 2012). Food and Agricultural Organization (FAO) report estimates that in Africa, deforestation accounts for nearly $70 \%$ of total emissions (FAO, 2005). Clearing tropical forests also destroy globally important carbon sinks that are currently sequestering $\mathrm{CO}_{2}$ from the atmosphere which are critical to future climate stabilization (Stephens et al., 2007).

Effective sequestration which is the net exclusion of carbon dioxide from the atmosphere and entrapment within long termed natural sinks is achieved by the presence and activities of plant species with life spans of not less than ten (10) years (SSSA, 2001). With this, estimates of carbon stocks and stock changes in tree biomass are necessary. Nigeria lack articulated data generation and banking in this area, hence stands to lose her natural carbon sinks. Above-ground biomass, below-ground biomass, dead wood, litter, and soil organic matter are the major carbon pools in any flora and fauna (Chavan and Rasal, 2012). Soil organic carbon is a large and active pool, containing roughly twice as much carbon as the atmosphere and 2.5 times as much as the biota and the amount of carbon sequestered at a site reflects the long-term balance between influx and efflux of carbon (SSSA, 2001).

Benue state $\left(7^{\circ} 43^{\prime} 50^{\prime \prime} \mathrm{N} 8^{\circ} 32^{\prime} 10^{\prime \prime} \mathrm{E}\right)$ has fourteen (14) forest reserves as part of her vegetative endowment. The primary and destructive activity going on at these sites is wood cutting/lumber business and this activity has become a threat to the existing biodiversity of the communities around the studied area. The culture of replanting is next to non-existent and with the establishment of industries as well as the existence of green house gas (GHG) generating activities; there is imminent risk exposure to the environment.

Guesstimates of carbon stocks and stock changes in tree biomass are essential for keying into the United Nations Framework Convention on Climate Change (UNFCCC) programme and as required by Kyoto Protocol as well as for carbon credit trading. This research is the first phase of assessment of carbon sequestration potential of tree species found within forest reserves and sites in Benue State.

\section{Methodology}

Sampling: Ten (10) sampling locations were identified and used in this investigation from two local government area namely: Gwer west and Makurdi. In Gwer west, Nagi was chosen as the sampling location due to its forest reserve with rural low emission area. Similarly, in Makurdi, Agan and Makurdi metropolis were chosen as sampling location with mild to heavy traffic emission areas. A total of four thousand two hundred (4200) trees belonging to twenty (20) different tree species were involved: Gmelina arborea, Tectona grandis, Anacardium occidentale L., Sarcocephalus latifolius, Anogeisus leiocarpa, Khaya senegalensis, Maranthes polyandra, Strychnos spinosa and Detarium microcarpum. Others are: Parkia biglobosa, Eucalyptus globules, Albizia lebbeck, Delonix regia, Schefflera actinophylla, Azadirachta Indica, Mangifera indica, Polyalthia longifolia, Ficus benjamina, Persea americana and Elaeis guineensis.

Analytical Method: Using non-destructive method, the mean above and below ground organic carbon (tones/tree) and total organic carbon of each species of tree was calculated. Carbon enters the soil as roots, litter, harvest residues, and animal manure. Carbon is stored primarily as soil organic matter (SOM) and this was determined by the Walkley-Black procedure (SSSA, 2001) and the carbon stock was calculated with the formula $=$ $\mathrm{C}(\%) / 100 \times$ soil bulk density $\times$ area $(1 \mathrm{ha}) \times$ soil depth (Figure 1).

\subsection{Diameter at Breast Height (DBH) and Tree Height (TH)}

DBH was calculated by measuring the tree Trunk Circumference at $1.3 \mathrm{~m}$ from the ground for each tree with circumferences greater than $10 \mathrm{~cm}$ while direct measurement neglecting height from ground was done for trees with circumference less or equal to $10 \mathrm{~cm}$ (Figure 2).

TH was obtained by direct measuring with surveyors' tape by climbing, aided by a pole and confirmation us- 
ing calculation with Thiodolite angle and sides calculation (Figure 3).

The angle between the tree top and eye view at breast height angle $(\alpha)$ is taken into consideration for tree height measurement and height of the tree is calculated. Considering the angle ACB between tree top and the distance (b) at the point of observer at $\mathrm{DBH}$, the tree height was calculated if $\alpha$ is the angle between eye view and top of the tree, a is the height of the tree in feet, c is the slope between tree and eye view, b is the distance in feet between tree and observer and $\mathrm{h}$ is height of horizontal plane of Thiodolite instrument, then the height of tree $(\mathrm{H})$ is calculated by the following formulae:

$$
\mathrm{H}=\mathrm{h}+\mathrm{b} \tan \alpha
$$

\subsection{Determination of Biomass and Carbon Dioxide Sequestered by Tree}

We adopted the method recommended by Chavan and Rasal (2010) with slight modification into five stages as follows:

1) Total (green) weight of tree based on tree species for tropical forest species, the weight of a tree is:

$\mathrm{W}=$ Above-ground weight of the tree in kilogram $(\mathrm{kg}) ; \mathrm{D}=$ Diameter of the trunk in inches; $\mathrm{H}=\mathrm{Height}$ of tree in Meters. Hence, for trees with $\mathrm{D} \leq 10: \mathrm{W}=0.25 \mathrm{D}^{2} \mathrm{H}$ or trees with $\mathrm{D} \geq 11: \mathrm{W}=0.15 \mathrm{D}^{2} \mathrm{H}$. The root system weighs about $20 \%$ as much as the above-ground weight of the tree. Therefore, to determine the total green weight of the tree, multiply the above-ground weight of the tree by $120 \%$.

2) Dry weight of tree based on an extension publication from the University of Nebraska (Chavan and Rasal, 2010).

The dry weight of the tree was calculated by multiplying the weight of the tree by $72.5 \%$.

3) Weight of carbon, The average carbon content is generally 50\% of the tree's total volume (DeWald et al., 2005).

Therefore, the weight of carbon in the tree was calculated by multiplying the dry weight of the tree by $50 \%$.

4) Weight of carbon dioxide sequestered $\left(\mathrm{CO}_{2}\right.$ is composed of one molecule of Carbon and 2 molecules of Oxygen and The atomic weight of Carbon is 12.001115; The atomic weight of Oxygen is 15.9994). Hence, weight of $\mathrm{CO}_{2}$ is $\mathrm{C}+(2 \mathrm{X} \mathrm{O})=43.999915$, while the ratio of $\mathrm{CO}_{2}$ to $\mathrm{C}$ is $43.999915 / 12.001115=3.6663$. Therefore, to determine the weight of carbon dioxide sequestered in the tree, we multiplied the Weight of carbon in the tree by 3.6663 (Birdsey, 1992).

5) Weight of $\mathrm{CO}_{2}$ sequestered in the tree per year was calculated by dividing the weight of carbon dioxide sequestered in the tree by the age of the tree.

Statistical analysis was carried out using Genstat sp 18 package. The procedures used include summary statistics and linear regression.

\section{Results and Discussion}

Assessment of carbon sequestration and storage potential of 4200 trees belonging to twenty (20) tropical tree species found within a local forest reserve and other sites in Makurdi Benue State of Nigeria was investigated. Carbon storage and sequestration potential of trees within Benue is presented from a rural traffic low emission area (Table 1), a mild to heavy traffic area (Table 2) and a heavy traffic high emission township (Tables 3-10), respectively. The relationship between dry weight of the studied trees and total sequestered $\mathrm{CO}_{2}$ is shown in Figure 4, while the relationship between heights of the studied trees to the total amount of $\mathrm{CO}_{2}$ sequestered (Figure 5), interprets the findings.

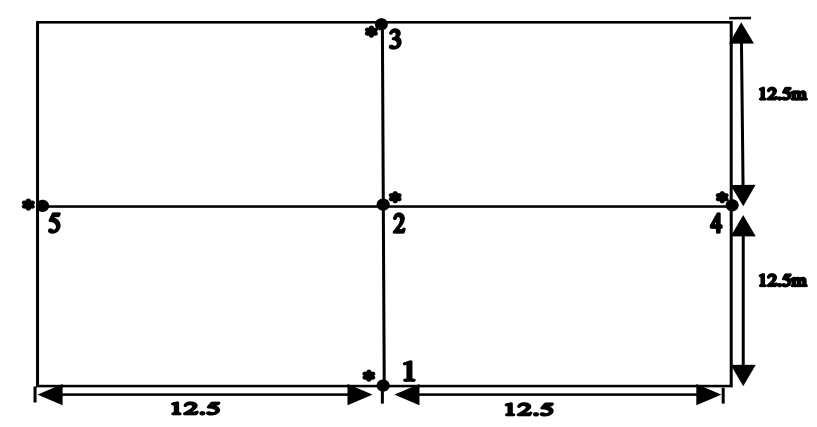

Figure 1. Soil sample collection method, * = Sampling point. 


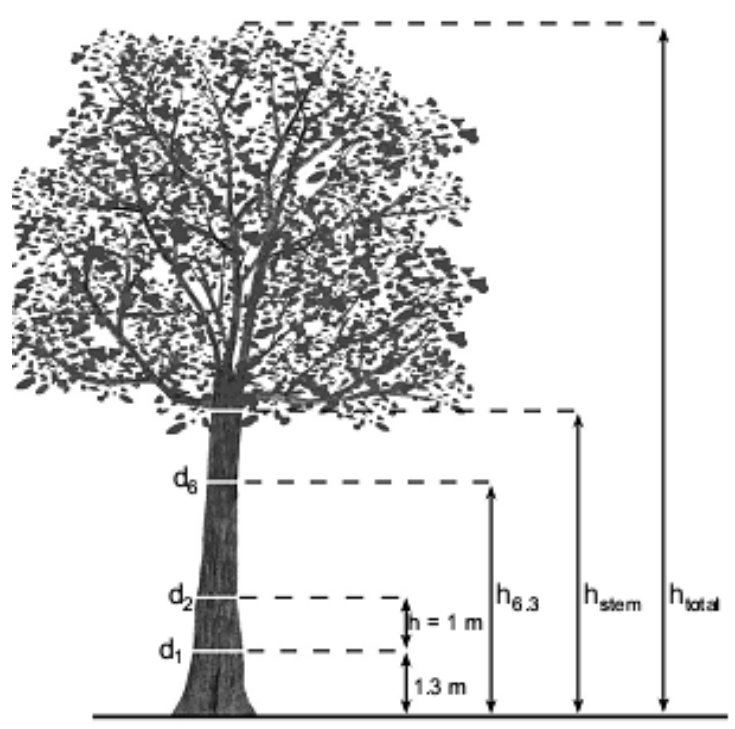

Figure 2. Diagrammatic representation of tree measurement methodology (Chavan, 2010).

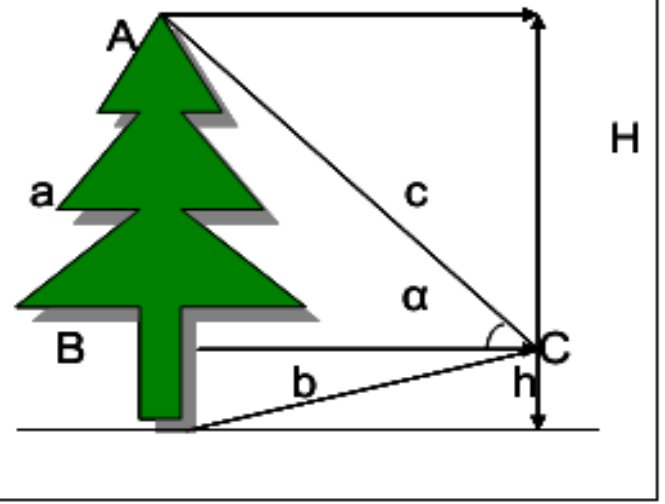

Figure 3. Tree height measurement by Thiodolite at DBH (SSSA, 2001).

Table 1. Assessment of tree species from Nagi, Gwer West (rural low emission area).

\begin{tabular}{|c|c|c|c|c|c|c|c|c|c|}
\hline \multirow[b]{2}{*}{$\mathrm{S} / \mathrm{N}$} & \multirow[b]{2}{*}{ Species } & \multirow[b]{2}{*}{ Number } & \multirow[b]{2}{*}{ TH (m) } & \multirow{2}{*}{$\begin{array}{c}\mathrm{DBH} \\
\text { (inches) }\end{array}$} & \multicolumn{5}{|c|}{ Weight from tree $(\mathrm{kg})$} \\
\hline & & & & & Total (green) & Dry & Carbon & $\begin{array}{c}\mathrm{CO}_{2} \\
\text { sequestered }\end{array}$ & $\begin{array}{c}\text { Average } \mathrm{CO}_{2} \\
\text { sequestered }\end{array}$ \\
\hline 1 & Gmelina arborea & 136 & 20.0 & 29.7 & $3,164.8$ & $1,912.0$ & 956.0 & $9,702.0$ & 71.3 \\
\hline 2 & Sarcocephalus latifolius & 68 & 6.9 & 25.1 & 779.4 & 470.9 & 235.5 & $2,390.7$ & 35.2 \\
\hline 3 & Parkia biglobosa & 12 & 12.0 & 21.5 & 998.5 & 603.2 & 301.6 & $3,050.6$ & 254.2 \\
\hline 4 & Anogeisus leiocarpa & 12 & 6.2 & 51.8 & $3,005.3$ & $1,815.7$ & 907.9 & $9,148.9$ & 762.4 \\
\hline \multirow[t]{2}{*}{5} & Tectona grandis & 248 & 10.0 & 21.7 & 844.5 & 510.2 & 255.1 & $2,589.6$ & 10.4 \\
\hline & Total & 476 & 55.1 & 149.8 & $8,792.5$ & $3,682.0$ & $2,656.1$ & $26,881.8$ & $1,133.5$ \\
\hline
\end{tabular}


Table 2. Assessment of tree species from Toll Gate, Makurdi-Lafia road (mild to heavy traffic area).

\begin{tabular}{|c|c|c|c|c|c|c|c|c|c|}
\hline \multirow[b]{2}{*}{$\mathrm{S} / \mathrm{N}$} & \multirow[b]{2}{*}{ Species } & \multirow[b]{2}{*}{ Number } & \multirow[b]{2}{*}{$\mathrm{TH}(\mathrm{m})$} & \multirow{2}{*}{$\begin{array}{c}\mathrm{DBH} \\
\text { (inches) }\end{array}$} & \multicolumn{5}{|c|}{ Weight from tree (kg) } \\
\hline & & & & & $\begin{array}{l}\text { Total } \\
\text { (green) }\end{array}$ & Dry & Carbon & $\begin{array}{c}\mathrm{CO}_{2} \\
\text { sequestered }\end{array}$ & $\begin{array}{c}\text { Average } \mathrm{CO}_{2} \\
\text { sequestered }\end{array}$ \\
\hline 1 & Tectona grandis & 156 & 10.4 & 25.1 & $1,181.3$ & 713.7 & 356.9 & $3,603.3$ & 23.1 \\
\hline 2 & Maranthes polyandra & 60 & 7.5 & 22.2 & 663.5 & 400.9 & 200.5 & $2,032.8$ & 33.9 \\
\hline 3 & Sarcocephalus latifolius & 4 & 8.2 & 19.1 & 539.0 & 449.2 & 224.5 & $1,645.1$ & 411.3 \\
\hline 4 & Strychnos spinosa & 4 & 6.9 & 15.0 & 279.5 & 168.8 & 84.4 & 853.8 & 213.5 \\
\hline 5 & Detarium microcarpum & 24 & 11.0 & 27.9 & $1,542.4$ & 931.8 & 465.9 & $4,708.9$ & 196.2 \\
\hline 6 & Gmelina arborea & 212 & 12.0 & 20.1 & 869.2 & 525.1 & 262.6 & $2,655.6$ & 12.5 \\
\hline \multirow[t]{2}{*}{7} & Anacardium occidentale L. & 8 & 10.0 & 44.0 & $3,476.9$ & $2,100.6$ & $1,050.3$ & $10,646.9$ & $1,330.9$ \\
\hline & TOTAL & 468.0 & 66.0 & 173.4 & $8,551.8$ & $5,290.1$ & $2,645.1$ & $26,146.4$ & $2,221.4$ \\
\hline
\end{tabular}

Table 3. Assessment of tree species from Makurdi local government township area (high emission area).

\begin{tabular}{|c|c|c|c|c|c|c|c|c|c|}
\hline \multirow[b]{2}{*}{$\mathrm{S} / \mathrm{N}$} & \multirow[b]{2}{*}{ Species } & \multirow[b]{2}{*}{ Number } & \multirow[b]{2}{*}{$\mathrm{TH}(\mathrm{m})$} & \multirow{2}{*}{$\begin{array}{c}\mathrm{DBH} \\
\text { (inches) }\end{array}$} & \multicolumn{5}{|c|}{ Weight from tree $(\mathrm{kg})$} \\
\hline & & & & & $\begin{array}{c}\text { Total } \\
\text { (green) }\end{array}$ & Dry & Carbon & $\begin{array}{c}\mathrm{CO}_{2} \\
\text { sequestered }\end{array}$ & $\begin{array}{c}\text { Average } \mathrm{CO}_{2} \\
\text { sequestered }\end{array}$ \\
\hline 1 & Eucalyptus globulus & 8 & 22.5 & 100.2 & $40,694.6$ & $24,566.7$ & $12,283.4$ & $124,233.1$ & $15,529.1$ \\
\hline 2 & Delonix regia & 368 & 7.3 & 47.9 & $3,002.3$ & $1,813.9$ & 907.0 & $9,211.1$ & 25.0 \\
\hline 3 & Albizia lebbeck & 48 & 8.1 & 48.8 & $3,443.9$ & $2,080.7$ & $1,040.4$ & $10,608.3$ & 221.0 \\
\hline 4 & Schefflera actinophylla & 156 & 10.0 & 41.9 & 3,152.6 & $1,904.7$ & 952.4 & $9,654.9$ & 61.9 \\
\hline 5 & Parkia biglobosa & 12 & 10.0 & 76.9 & $10,630.7$ & $6,422.7$ & $3,211.4$ & $32,521.6$ & $2,710.1$ \\
\hline 6 & Gmelina arborea & 32 & 10.3 & 77.1 & $10,956.0$ & 6,619.3 & $3,309.7$ & $33,671.7$ & $1,052.2$ \\
\hline 7 & Azadirachta Indica & 20 & 15.2 & 81.2 & $18,037.0$ & $10,897.6$ & $5,448.8$ & $55,115.7$ & $2,755.8$ \\
\hline 8 & Mangifera indica & 24 & 14.0 & 106.1 & $28,362.0$ & $17,135.2$ & $8,567.6$ & $86,671.8$ & $3,611.3$ \\
\hline 9 & Polyalthia longifolia & 16 & 10.8 & 18.8 & 683.5 & 413.0 & 206.5 & $2,099.2$ & 131.2 \\
\hline \multirow[t]{2}{*}{10} & Ficus benjamina & 32 & 6.8 & 35.7 & $1,560.9$ & 943.1 & 471.6 & $4,766.1$ & 148.9 \\
\hline & TOTAL & 716 & 115.0 & 634.6 & $120,523.5$ & $72,796.9$ & $36,398.8$ & $368,553.5$ & $26,246.5$ \\
\hline
\end{tabular}

Table 4. Assessment of tree species from Wurukum Round About to School of Remedial Studies Junction.

\begin{tabular}{|c|c|c|c|c|c|c|c|c|c|}
\hline \multirow[b]{2}{*}{$\mathrm{S} / \mathrm{N}$} & \multirow[b]{2}{*}{ Species } & \multirow[b]{2}{*}{ Number } & \multirow[b]{2}{*}{$\mathrm{TH}(\mathrm{m})$} & \multirow[b]{2}{*}{$\begin{array}{c}\mathrm{DBH} \\
\text { (inches) }\end{array}$} & \multicolumn{5}{|c|}{ Weight from tree (kg) } \\
\hline & & & & & $\begin{array}{c}\text { Total } \\
\text { (green) }\end{array}$ & Dry & Carbon & $\begin{array}{c}\mathrm{CO}_{2} \\
\text { sequestered }\end{array}$ & $\begin{array}{c}\text { Average } \mathrm{CO}_{2} \\
\text { sequestered }\end{array}$ \\
\hline 1 & Flambouyant & 140 & 9.3 & 51.4 & $4,443.2$ & $2,684.5$ & $1,342.3$ & $13,512.3$ & 96.5 \\
\hline 2 & Umbrella tree & 60 & 10.2 & 39.1 & $2,806.0$ & $1,695.3$ & 847.7 & $8,575.8$ & 142.9 \\
\hline 3 & Albizia & 8 & 18.5 & 87.7 & $25,618.4$ & $15,477.7$ & $7,738.9$ & $78,251.1$ & $9,781.4$ \\
\hline 4 & Gmeliana & 28 & 19.9 & 89.5 & $28,644.6$ & $17,306.1$ & $8,653.1$ & $87,663.4$ & $3,130.8$ \\
\hline 5 & Eucalyptus & 4 & 25.0 & 65.2 & $19,104.5$ & $11,542.3$ & $5,771.2$ & $58,446.0$ & $14,611.5$ \\
\hline 6 & Neem & 20 & 15.8 & 61.0 & $10,563.5$ & $6,382.1$ & 3191.1 & $32,332.3$ & $1,616.6$ \\
\hline 7 & Pea & 16 & 6.5 & 55.1 & $3,556.4$ & $2,148.7$ & $1,074.4$ & $10,852.7$ & 678.3 \\
\hline 8 & Cocoanut tree & 8 & 22.5 & 57.6 & $13,455.0$ & $8,129.1$ & $4,064.6$ & $41,053.2$ & $5,131.7$ \\
\hline 9 & Mango & 12 & 16.7 & 90.2 & $24,422.7$ & $14,755.4$ & $7,377.7$ & $74,722.1$ & 6226.8 \\
\hline 10 & Cashew & 16 & 19.5 & 119.3 & $49,945.2$ & $30,175.2$ & $15,087.6$ & $152,628.2$ & 9,539.3 \\
\hline \multirow[t]{2}{*}{11} & Masquerade & 12 & 18.3 & 40.1 & $5,304.4$ & $3,204.8$ & $1,602.4$ & $16,183.0$ & $1,348.9$ \\
\hline & TOTAL & 324 & 182.2 & 756.2 & $187,863.9$ & $113,501.2$ & $56,751.0$ & $574,220.1$ & $52,304.7$ \\
\hline
\end{tabular}


Table 5. Assessment of tree species from Wurukum Round about to Low level Round about.

\begin{tabular}{|c|c|c|c|c|c|c|c|c|c|}
\hline \multirow{2}{*}{$\mathrm{S} / \mathrm{N}$} & \multirow{2}{*}{ Species } & \multirow{2}{*}{ Number } & \multirow{2}{*}{$\begin{array}{l}\mathrm{TH} \\
(\mathrm{m})\end{array}$} & \multirow{2}{*}{$\begin{array}{c}\text { DBH } \\
\text { (inches) }\end{array}$} & \multicolumn{5}{|c|}{ Weight from tree $(\mathrm{kg})$} \\
\hline & & & & & $\begin{array}{c}\text { Total } \\
\text { (green) }\end{array}$ & Dry & Carbon & $\begin{array}{c}\mathrm{CO}_{2} \\
\text { sequestered }\end{array}$ & $\begin{array}{c}\text { Average } \mathrm{CO}_{2} \\
\text { sequestered }\end{array}$ \\
\hline 1 & Delonix regia & 352 & 7.1 & 48.6 & $3,033.3$ & $1,832.6$ & 916.3 & $9,222.5$ & 26.2 \\
\hline 2 & Schefflera actinophylla & 12 & 8.0 & 30.1 & $1,302.3$ & 786.8 & 393.4 & $3,986.1$ & 332.2 \\
\hline 3 & Azadirachta Indica & 28 & 17.0 & 125.3 & $48,038.4$ & $29,023.0$ & $14,511.5$ & $146,781.2$ & $5,242.2$ \\
\hline 4 & Persea americana & 12 & 11.0 & 81.9 & $13,263.6$ & $8,013.0$ & 4006.5 & $40,577.0$ & $3,381.4$ \\
\hline 5 & Gmelina arborea & 12 & 20.0 & 128.8 & $59,730.3$ & $36,087.0$ & $18,043.5$ & $182,465.6$ & $15,205.5$ \\
\hline 6 & Mangifera indica & 8 & 11.5 & 85.2 & $15,027.9$ & $9,079.4$ & $4,539.7$ & $45,908.8$ & $5,738.6$ \\
\hline 7 & Anacardium occidentale $L$ & 16 & 14.3 & 87.7 & $19,733.1^{`}$ & $11,922.0$ & $5,961.0$ & $60,486.0$ & $3,780.4$ \\
\hline \multirow[t]{2}{*}{8} & Eucalyptus globulus & 8 & 16.0 & 72.7 & $15,211.2$ & $9,190.1$ & 4595.1 & $46,505.9$ & $5,813.2$ \\
\hline & TOTAL & 448 & 104.9 & 660.3 & $175,340.1$ & $105,933.9$ & $52,967.0$ & $535,933.1$ & $39,519.7$ \\
\hline
\end{tabular}

Table 6. Assessment of tree species from low level Round about to high level Round about in Makurdi.

\begin{tabular}{|c|c|c|c|c|c|c|c|c|c|}
\hline \multirow[b]{2}{*}{$\mathrm{S} / \mathrm{N}$} & \multirow[b]{2}{*}{ Species } & \multirow[b]{2}{*}{ Number } & \multirow[b]{2}{*}{$\mathrm{TH}(\mathrm{m})$} & \multirow[b]{2}{*}{$\begin{array}{c}\mathrm{DBH} \\
\text { (inches) }\end{array}$} & \multicolumn{5}{|c|}{ Weight from tree $(\mathrm{kg})$} \\
\hline & & & & & $\begin{array}{c}\text { Total } \\
\text { (green) }\end{array}$ & Dry & Carbon & $\begin{array}{c}\mathrm{CO}_{2} \\
\text { sequestered }\end{array}$ & $\begin{array}{c}\text { Average } \mathrm{CO}_{2} \\
\text { sequestered }\end{array}$ \\
\hline 1 & Delonix regia & 440 & 5.8 & 49.9 & $2,578.1$ & $1,557.6$ & 778.8 & $7,942.3$ & 18.1 \\
\hline 2 & Schefflera actinophylla & 24 & 9.7 & 42.6 & $3,070.4$ & $1,909.2$ & 954.6 & $9,680.8$ & 403.4 \\
\hline 3 & Polyalthia longifolia & 12 & 5.3 & 18.0 & 312.2 & 188.6 & 94.3 & 944.4 & 78.7 \\
\hline 4 & Albizia lebbeck & 16 & 11.3 & 58.9 & $7,023.2$ & $4,243.2$ & $2,121.6$ & $21,559.0$ & $1,347.4$ \\
\hline 5 & Mangifera indica & 4 & 12.0 & 100.2 & $21,704.2$ & $13,112.9$ & $6,556.5$ & $66,257.6$ & $16,564.4$ \\
\hline \multirow[t]{2}{*}{6} & Persea americana & 12 & 8.0 & 40.1 & $2,315.8$ & $1,399.1$ & 699.6 & $7,074.5$ & 589.5 \\
\hline & TOTAL & 508 & 51.4 & 309.7 & $37,003.9$ & $22,410.6$ & $11,205.4$ & $113,458.6$ & $19,001.5$ \\
\hline
\end{tabular}

Table 7. Assessment of tree species from high level to B-Division Junction in Makurdi.

\begin{tabular}{|c|c|c|c|c|c|c|c|c|c|}
\hline \multirow[b]{2}{*}{$\mathrm{S} / \mathrm{N}$} & \multirow[b]{2}{*}{ Species } & \multirow[b]{2}{*}{ Number } & \multirow[b]{2}{*}{ TH (m) } & \multirow[b]{2}{*}{$\begin{array}{c}\mathrm{DBH} \\
\text { (inches) }\end{array}$} & \multicolumn{5}{|c|}{ Weight from tree (kg) } \\
\hline & & & & & $\begin{array}{l}\text { Total } \\
\text { (green) }\end{array}$ & Dry & Carbon & $\begin{array}{c}\mathrm{CO}_{2} \\
\text { sequestered }\end{array}$ & $\begin{array}{c}\text { Average } \mathrm{CO}_{2} \\
\text { sequestered }\end{array}$ \\
\hline 1 & Schefflera actinophylla & 144 & 8.3 & 41.5 & $2,578.9$ & $1,558.1$ & 779.1 & $7,861.3$ & 54.6 \\
\hline 2 & Delonix regia & 132 & 6.6 & 63.4 & $4,800.4$ & $2,900.3$ & $1,450.2$ & $14,589.5$ & 110.5 \\
\hline 3 & Gmelina arborea & 8 & 16.0 & 168.6 & $81,822.0$ & $49,434.2$ & $24,717.1$ & $250,123.4$ & $31,265.4$ \\
\hline \multirow[t]{2}{*}{4} & Elaeis guineensis & 4 & 15.0 & 191.4 & $98,935.5$ & $59,773.5$ & $29,886.8$ & 302,199.9 & $75,550.0$ \\
\hline & TOTAL & 288 & 45.9 & 464.9 & $188,135.8$ & $113,666.1$ & $56,833.2$ & $574,308.0$ & $78,841.5$ \\
\hline
\end{tabular}


Table 8. Assessment of tree species from low level Round about to State house in Makurdi.

\begin{tabular}{|c|c|c|c|c|c|c|c|c|c|}
\hline \multirow[b]{2}{*}{$\mathrm{S} / \mathrm{N}$} & \multirow[b]{2}{*}{ Species } & \multirow[b]{2}{*}{ Number } & \multirow{2}{*}{$\begin{array}{l}\mathrm{TH} \\
(\mathrm{m})\end{array}$} & \multirow{2}{*}{$\begin{array}{c}\mathrm{DBH} \\
\text { (inches) }\end{array}$} & \multicolumn{5}{|c|}{ Weight from tree (kg) } \\
\hline & & & & & $\begin{array}{l}\text { Total } \\
\text { (green) }\end{array}$ & Dry & Carbon & $\begin{array}{c}\mathrm{CO}_{2} \\
\text { sequestered }\end{array}$ & $\begin{array}{c}\text { Average } \mathrm{CO}_{2} \\
\text { sequestered }\end{array}$ \\
\hline 1 & Anacardium occidentale L. & 4 & 5.0 & 31.9 & 916.5 & 553.7 & 276.85 & $2,798.2$ & 699.6 \\
\hline 2 & Delonix regia & 440 & 6.1 & 62.5 & $4,257.6$ & $2,572.3$ & $1,286.2$ & $13,104.2$ & 29.8 \\
\hline 3 & Gmelina arborea & 28 & 10.3 & 60.6 & $6,805.3$ & $4,111.5$ & $2,055.8$ & $20,801.8$ & 742.9 \\
\hline 4 & Mangifera indica & 12 & 25.0 & 250.8 & $282,956.0$ & $170,953.0$ & $85,476.5$ & $864,797.3$ & $72,066.4$ \\
\hline \multirow[t]{2}{*}{5} & Persea americana & 4 & 25.0 & 191.4 & $164,892.0$ & $99,622.5$ & $49,811.3$ & $503,666.6$ & $125,916.7$ \\
\hline & TOTAL & 488 & 71.4 & 597.2 & $459,827.4$ & $107,031.0$ & $138,906.7$ & $1,405,168.1$ & $199,455.4$ \\
\hline
\end{tabular}

Table 9. Assessment of tree species from Wurukum Round about to Guarantee Trust Bank Junction.

\begin{tabular}{|c|c|c|c|c|c|c|c|c|c|}
\hline \multirow[b]{2}{*}{$\mathrm{S} / \mathrm{N}$} & \multirow[b]{2}{*}{ Species } & \multirow[b]{2}{*}{ Number } & \multirow[b]{2}{*}{$\mathrm{TH}(\mathrm{m})$} & \multirow{2}{*}{$\begin{array}{c}\text { DBH } \\
\text { (inches) }\end{array}$} & \multicolumn{5}{|c|}{ Weight from tree (kg) } \\
\hline & & & & & $\begin{array}{l}\text { Total } \\
\text { (green) }\end{array}$ & Dry & Carbon & $\begin{array}{c}\mathrm{CO}_{2} \\
\text { sequestered }\end{array}$ & $\begin{array}{c}\text { Average } \mathrm{CO}_{2} \\
\text { sequestered }\end{array}$ \\
\hline 1 & Albizia lebbeck & 8 & 7.0 & 55.3 & $3,855.6$ & $2,329.4$ & $1,164.7$ & $11,772.5$ & $1,471.6$ \\
\hline 2 & Delonix regia & 388 & 5.1 & 47.6 & $2,064.8$ & $1,247.5$ & 623.8 & $6,354.8$ & 16.4 \\
\hline 3 & Mangifera indica & 4 & 10.0 & 50.1 & $4,521.8$ & $2,731.9$ & $1,366.0$ & $13,803.7$ & $3,450.9$ \\
\hline 4 & Elaeis guineensis & 16 & 8.0 & 129.7 & $24,233.5$ & $14,641.0$ & $7,320.5$ & $74,009.8$ & $4,625.6$ \\
\hline 5 & Persea americana & 4 & 4.0 & 40.1 & $1,157.9$ & 699.6 & 349.8 & $3,537.3$ & 884.3 \\
\hline 6 & Azadirachta Indica & 4 & 13.0 & 115.3 & $31,096.3$ & $18,787.0$ & $9,393.5$ & $95,043.2$ & $23,760.8$ \\
\hline 7 & Eucalyptus globulus & 20 & 25.0 & 108.3 & $52,742.9$ & $31,865.5$ & $15,932.8$ & $161,256.1$ & $8,062.8$ \\
\hline 8 & Parkia biglobosa & 16 & 12.8 & 94.0 & $20,269.1$ & $12,245.9$ & $6,123.0$ & $62,199.2$ & $3,887.5$ \\
\hline \multirow[t]{2}{*}{9} & Schefflera actinophylla & 20 & 9.2 & 47.6 & $3,754.5$ & $2,268.3$ & $1,134.2$ & $11,463.6$ & 573.2 \\
\hline & TOTAL & 480 & 94.1 & 688 & $143,696.4$ & $86,816.1$ & $43,408.3$ & $439,440.2$ & $46,213.1$ \\
\hline
\end{tabular}

Table 10. Assessment of tree species from Police headquarters Round about to Federal Medical Centre in Makurdi.

\begin{tabular}{|c|c|c|c|c|c|c|c|c|c|}
\hline \multirow[b]{2}{*}{$\mathrm{S} / \mathrm{N}$} & \multirow[b]{2}{*}{ Species } & \multirow[b]{2}{*}{ Number } & \multirow[b]{2}{*}{$\mathrm{TH}(\mathrm{m})$} & \multirow{2}{*}{$\begin{array}{l}\mathrm{DBH} \\
\text { (inches) }\end{array}$} & \multicolumn{5}{|c|}{ Weight from tree (kg) } \\
\hline & & & & & $\begin{array}{l}\text { Total } \\
\text { (green) }\end{array}$ & Dry & Carbon & $\begin{array}{c}\mathrm{CO}_{2} \\
\text { sequestered }\end{array}$ & $\begin{array}{c}\text { Average } \mathrm{CO}_{2} \\
\text { sequestered }\end{array}$ \\
\hline 1 & Delonix regia & 172 & 5.8 & 47.1 & $2,308.4$ & $1,394.7$ & 697.4 & $7,076.0$ & 41.1 \\
\hline 2 & Albizia lebbeck & 92 & 8.6 & 53.6 & $4,447.9$ & $2,687.3$ & $1,343.7$ & $13,587.7$ & 147.7 \\
\hline 3 & Mangifera indica & 4 & 15.0 & 200.5 & $108,523.0$ & $65,566.2$ & $32,783.1$ & $331,618.9$ & $82,904.7$ \\
\hline 4 & Gmelina arborea & 4 & 12.0 & 62.7 & $8,477.5$ & $5,121.5$ & $2,560.8$ & 25,943.9 & $6,486.0$ \\
\hline 5 & Schefflera actinophylla & 36 & 6.1 & 40.1 & $1,768.7$ & $1,068.6$ & 534.3 & $5,394.3$ & 149.8 \\
\hline 6 & Persea americana & 12 & 7.5 & 69.2 & $6,458.3$ & $3,901.8$ & $1,950.9$ & $19,751.2$ & $1,645.9$ \\
\hline \multirow[t]{2}{*}{7} & Azadirachta Indica & 8 & 9.0 & 60.1 & $5,859.3$ & $3,539.9$ & $1,770.0$ & $17,877.7$ & $2,234.7$ \\
\hline & TOTAL & 328 & 64.00 & 533.30 & $137,843.10$ & $83,280.00$ & $41,639.30$ & $421,249.70$ & $93,609.90$ \\
\hline
\end{tabular}




\section{Discussion}

The total amount of $\mathrm{CO}_{2}$ sequestered at the studied areas is presented from the lowest locations as; Low level roundabout to State House roundabout (1,405,168.1 kg); High level to B-Division Junction (574,308.0 kg); Wurukum round about to SRS Junction $(574,220.1 \mathrm{~kg})$; Wurukum round about to Low level roundabout (535,933.1 kg); Wurukum round about to GT Bank Junction (439,440.2 kg); Police headquarters round about to Federal Medical Center (421,249.7kg); Benue State University Teaching Hospital to Wurukum round about (368553.5 kg); Nagi-Naka, Gwer West Local Government Area (rural traffic low emission area 26881.8 kg); Toll Gate, Agan Village, Makurdi-Lafia road (mild to heavy traffic area $26146.4 \mathrm{~kg}$ ); Low level round about to High level round about (113,458.6 kg).

The relationship between the dry weight of trees and total sequestered $\mathrm{CO}_{2}$ in various locations within areas under investigation is shown in Figure 4. There is observed much higher level of $\mathrm{CO}_{2}$ sequestered in location 8 , with a value of $1,405,168.1 \mathrm{~kg}$. This may be connected to the nature, the age and specie of the trees, and the location as is corroborated by earlier works, with dominant species of sequestered $\mathrm{CO}_{2}$ at the site being Mangifera indica and Persea americana. The observed high value may be related to their broad leaves and thick vegetative cover resulting from the natural cluster, especially the Persea americana. This area stretches from low level round about to State House round about designated as part of "high traffic area”. Figure 1 also presents a generally observed trend that there is a progressively direct relationship between the dry weight of trees and the sequestered $\mathrm{CO}_{2}$, as represented by trees on the plot and the corresponding rectangles (Gibbs et al., 2007).

From the Tables, it is observed that trees species like Anogeisus leiocarpa, Mangifera indica, Parkia biglobosa, Azadirachta Indica, Anacardium occidentale, Eucalyptus globulus and Elaeis guineensis have more amount of carbon dioxide sequestered in them within the township area. While within the low emission area like Nagi, Naka, Anogeisus leiocarpa recorded the highest sequestration of $\mathrm{CO}_{2}$ and Tectona grandis with the lowest (762.4 kg and $10.4 \mathrm{~kg}$ ) respectively. For the Toll Gate area which is a medium emission area, Anacardium occidentale recorded the highest with Gmelina arborea having the lowest (1,330.9 $\mathrm{Kg}$ and $12.5 \mathrm{~kg})$ respectively. Meanwhile, along Gboko road, Mangifera indica has the highest and Schefflera actinophylla the lowest (3611.3 $\mathrm{kg}$ and $61.9 \mathrm{~kg}$ ) respectively.

Along Makurdi-Lafia road, Eucalyptus globulus recorded the highest with Delonix regia having the lowest (14611.5 kg and $96.5 \mathrm{~kg}$ ). From Wurukum round about to low level round about, a total amount of 535,933.1 kg of $\mathrm{CO}_{2}$ was sequestered by the trees which are relatively smaller than that obtained along location 8 with $\mathrm{Gme-}$ lina arborea absorbing the highest (182,465.6 kg) and the Schefflera actinophylla having the lowest (3,986.1 kg).

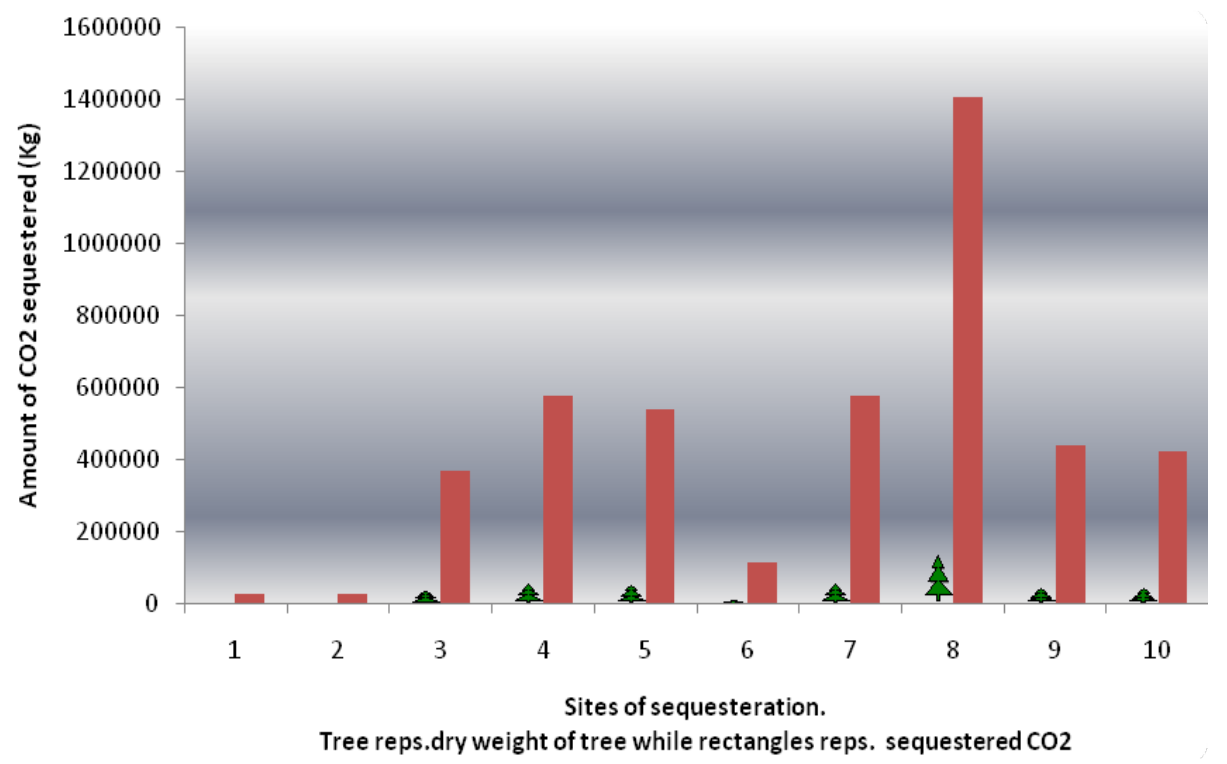

Figure 4. Relationship between dry weight and total sequestered $\mathrm{CO}_{2}$ of a tree. Keys: 1. reps. Nagi.; 2. reps. Toll gate.; 3. reps. BSUTH to Wurukum; 4. reps. Wurukum to SRS; 5. reps. Wurukum to Low level; 6. reps. Low level to High level; 7. reps. High level to B-Div; 8. reps. Low level to State House; 9. reps. Wurukum to GT Bank; 10. reps. Police hdqrt to F. M. C. 


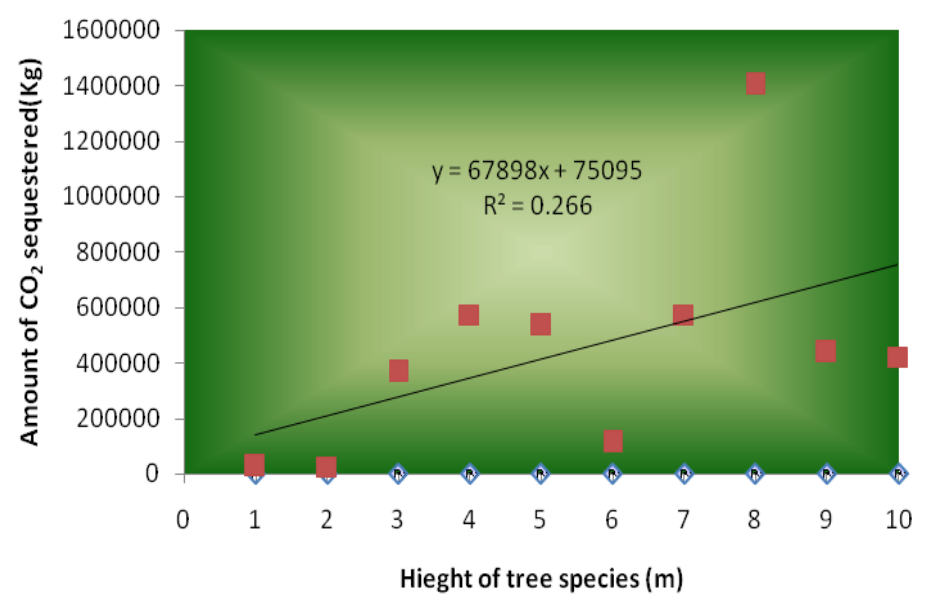

Figure 5. Relationship between height of tree against total amount of $\mathrm{CO}_{2}$ sequestered. Triangle reps. Tree height while squares reps. Amt. Of $\mathrm{CO}_{2}$ sequestered.

This observed general low absorption along location 5 can be attributed to the relatively scanty number of trees planted as well as the non existence of species like Mangifera indica with broad leaves and consequent thick vegetative cover, having known that thickness of cover and area of cover influences amount of absorbed $\mathrm{CO}_{2}$ by trees. In contrast to the observed low sequestration by the Schefflera actinophylla, the Delonix regia with a total no of 352 sequestered only 9,222.5. This brings to light the fact on individual basis, Schefflera actinophylla with individual sequestration of $332.2 \mathrm{~kg}$, is a better candidate for sequestration than Delonix regia with individual sequestration of $26.2 \mathrm{~kg}$. This substantiates the fact that the broader the leaves, the thicker the crown cover and consequently the denser the cluster; the more $\mathrm{CO}_{2}$ sequestered by trees, this aligns with earlier reported works (Rowntree and Nowak, 1991).

Figure 5 in trying to establish a relationship $(\mathrm{P}>0.05)$ between tree height and $\mathrm{CO}_{2}$ sequestration, show that there is no significant direct relationship between tree height and $\mathrm{CO}_{2}$ sequestration although the height of a tree contributes to the volume of the tree which can be related to the mass and consequently the relative $\mathrm{CO}_{2}$ sequestration potential of the tree (Rowntree, 1984). This is buttressed by the $r^{2}$ value of 0.266 . Noting that the closer the $r^{2}$ value to whole number 1 , the more directly the relationship exists between the factors and more perfect the line of best fit. The gradient of the scattered plot is 67898 and equation for the graph is $y=67898 x+75095$. The high value of the gradient is also an indication of the deviation from a linear best fit linear regression.

\section{Conclusion}

This preliminary evaluation strategically positions (guinea savanna) as found in Benue State in line for UNFCCC and Kyoto carbon credit trading while substantiating the importance of preserving our indigenous forest reserves. This is because recent importance has been attached to emissions reductions from tropical deforestation in future climate change policy (Rowntree, 1984). The United Nations Framework Convention on Climate Change recently agreed to study and consider a new initiative, led by forest-rich developing countries, that calls for economic incentives to help facilitate reductions in emissions from deforestation in developing countries (REDD). This is a proposal to provide financial incentives to help developing countries voluntarily reduce national deforestation rates and associated carbon emissions below a baseline.

This work has brought to bear the fact that $\mathrm{CO}_{2}$ emission within our local environment can be effectively sequestered by planted trees more so the landscaping of our major intra city motor ways by planting of mostly broad leaved trees will to a great extent mitigate against expunged $\mathrm{CO}_{2}$ gases from converted $\mathrm{CO}$ gases emitted from automobiles with consequent reduction in health and environmental related issues.

The result has also presented a guide on the type of tree species that ought to be planted for the sequestration of $\mathrm{CO}_{2}$ and $\mathrm{CO}$ gases for effective sequestration purpose. Such trees as Mango, Umbrella tree and other broad leaved species can be planted 20 or more meters away from the pedestal path along our city ways. These species of trees will not only aid in $\mathrm{CO}_{2}$ sequestration but also provide adequate shade and food for the populace. 


\section{References}

Birdsey, R. A. (1992). Carbon Storage and Accumulation in United States Forest Ecosystems, General Technical Report W0-59. Radnor, PA: United States Department of Agriculture Forest Service, Northeastern Forest Experiment Station. http://www.ilea.org/birdsey/fcarbon_index.html\#toc

Chavan, B. L., \& Rasal, G. B. (2010). Sequestered Standing Carbon Stock in Selective Tree Species Grown in University Campus at Aurangabad, Maharashtra, India. International Journal of Engineering Science and Technology, 2, 3003-3007.

Chavan, B., \& Rasal, G. (2012). Total Sequestered Carbon Stock of Mangifera indica. Journal of Environment and Earth Science, 2.

DeWald, S., Josiah, S., \& Erdkamp, B. (2013). Heating With Wood: Producing, Harvesting and Processing Firewood. University of Nebraska-Lincoln Extension, Institute of Agriculture and Natural Resources. http://www.ianrpubs.unl.edu/epublic/live/g1554/build/g1554.pdf

Dombro, D. B. (2006). How Much Carbon Does a Tropical Tree Sequester. http://www.tree-nation.com

FAO (2005). State of the Worlds Forests 2005. Rome: FAO.

FAO (Food and Agricultural Organization of the United Nations) (2005). FAO Statistical Database 2005. http://faostat.fao.org

FDF (2001). Forestry Outlook Study for African Country Report Nigeria. Prepared by Aruofor, R. Federal Department of Forestry.

Gibbs, H. K., Brown, S., Niles, J. O., \& Foley, J. A. (2007). Monitoring and Estimating Tropical Forest Carbon Stocks: Making REDD a Reality. Environmental Research Letters, 2, Article ID: 045023.

Guendehou, G. H. S., Lehtonen, A., Moudachirou, M., Mäkipää, R., \& Sinsin, B. (2012). Stem Biomass and Volume Models of Selected Tropical Tree Species in West Africa. Southern Forests, 74, 77-88.

http://www.ncsec.org/cadre2/team18 2/students/helpCalcCO2.htm

IPCC (2001). Climate Change 2001: The Scientific Basis. IPCC Third Assessment Report, Working Group I, Technical Summary. Cambridge: Cambridge University Press.

Nelson, D. W., \& Sommers, L. E. (1996). Total Carbon, Organic Carbon and Organic Matter. In A. L. Sparks, P. A. Page, R. H. Helmke, P. N. Loeppert, M. A. Soltanpour, C. T. Tabatabai, M. E. Johnson (Eds.), Methods of Soil Analysis. Part 3-Chemical Methods Soil Sci Soc Am Book Ser 5 (pp. 1961-2010). Madison, WI: SSSA.

Oguntala, A. B. (1995). Effect of Fuelwood Collection and Logging on Environment by 268 UNESCO MAB Regional Training Workshop, Akure, 23-26 July 1995.

Rowntree, R. A. (1984). Forest. Canopy Cover and Land Use in Four Eastern United States Cities. Urban Ecology, 8, 55-67.

Rowntree, R. A., \& Nowak, D. J. (1991). Quantifyingtherol Eofurbanforestsi N Removing Atmospheric Carbon Dioxide. Journal of Arboriculture, 17, 269-275.

Stephens, B. S. et al. (2007). Weak Northern and Strong Tropical Land Carbon Uptake from Vertical Profiles of Atmospheric $\mathrm{CO}_{2}$. Science, 316, 1732-1735.

Tagupa C., Lopez, A., \& Caperida, F., Pamunag, G., \& Luzada, A. (2010). Carbon Dioxide $\left(\mathrm{Co}_{2}\right)$ Sequestration Capacity of Tampilisan Forest. E-International Scientific Research Journal, 2.

www.shodor.org/....dhi/weightree/science2-content.html Accessed on 25th November, 2013. 